\title{
Investigation of the Movement of Cement Clinker Particles in the Screw Body of a Rotary Kiln for Its Making
}

\author{
A. N. Sekisov", ${ }^{1, *}$ G. V. Serga ${ }^{2}$, D. A. Gura ${ }^{3}$, A. A. Savenko ${ }^{1}$, V. P. Danko ${ }^{4}$ \\ ${ }^{1}$ Department of Technology, Organization, Construction Economics and Real Estate Management, Institute of Construction \\ and Transport Infrastructure, Kuban State Technological University, Krasnodar, Moscovskaya Str, 2, Krasnodar Region, Russia \\ ${ }^{2}$ Department of Descriptive Geometry and Graphics, Faculty of Architecture and Civil Engineering, Kuban State Agrarian \\ University, Krasnodar, Kalinina Str., 13, Krasnodar Region, Russia \\ ${ }^{3}$ Department of Cadastre and Geoengineering, Institute of Construction and Transport Infrastructure, Kuban State \\ Technological University, Krasnodar, Moscovskaya Str, 2, Krasnodar Region, Russia \\ ${ }^{4}$ Department of Production of Building Structures and Structural Mechanics, Institute of Construction and Transport \\ Infrastructure, Kuban State Technological University, Krasnodar, Moscovskaya Str, 2, Krasnodar Region, Russia
}

Received March 26, 2021; Revised May 26, 2021; Accepted October 17, 2021

\section{Cite This Paper in the following Citation Styles}

(a): [1] A. N. Sekisov, G. V. Serga, D. A. Gura, A. A. Savenko, V. P. Danko, "Investigation of the Movement of Cement Clinker Particles in the Screw Body of a Rotary Kiln for Its Making", Civil Engineering and Architecture, Vol. 9, No. 6, pp. 2098-2104, 2021. DOI: 10.13189/cea.2021.090637.

(b): A. N. Sekisov, G. V. Serga, D. A. Gura, A. A. Savenko, V. P. Danko (2021). Investigation of the Movement of Cement Clinker Particles in the Screw Body of a Rotary Kiln for Its Making. Civil Engineering and Architecture, 9(6), 2098-2104. DOI: 10.13189/cea.2021.090637.

Copyright $\mathrm{C} 2021$ by authors, all rights reserved. Authors agree that this article remains permanently open access under the terms of the Creative Commons Attribution License 4.0 International License

\begin{abstract}
The possibility of improving the technical and economic indicators of the technological process and equipment for the cement clinker making in rotary kilns by replacing the inclined cylindrical bodies of rotary kilns with screw bodies mounted horizontally is shown. The kinematics of motion of clinker particles in the screw body of a rotary kiln for the cement clinker making is analyzed and mathematical models of their motion inside the screw bodies are proposed. Examples of the created spatial forms of screw bodies of rotating furnaces with the use of the program complex "Compass-3D" are shown. Technical solutions protected by patents of the Russian Federation [1-9] are offered, and designs of bodies of the rotating furnaces are shown. The analytical study of the motion of the particles of cement raw mass is presented in the article, and output dependence to determine their velocity in a screw body of the rotary kiln have particular scientific and practical importance. In such furnaces, technical and economic advantages are achieved due to the longitudinal movement of the sludge pellets in a furnace for cement clinker making, the horizontal arrangement of the rotary kiln body and their counter flows, due to the fact that inside
\end{abstract}

the entire length of the screw body, formed of a screw surface and helical grooves, which provide not only the movement of granules in the sludge, but also contribute to the intensification of the interaction of sludge granules for cement clinker making with each other and with the walls of the body, and this allows not only influencing the nature of the movement of sludge granules for the cement clinker making and expanding the technological capabilities, increasing the intensity of heat exchange, simplifying the operation of the rotary kiln at its horizontal location, expanding the technological capabilities, but also reducing the dimensions of the furnaces in length and their weight.

Keywords Screw Body, Screw Lines, Screw Surfaces

\section{Introduction}

In the cement production in the firing of sludge for the cement clinker making they use powerful rotary kilns. 
Only in the last 20 years in the cement industry, the capacity of a single fired unit has increased by 5 times. The disadvantage of such rotary kilns for firing sludge in the cement clinker making is a need to create an incline in the body for transporting sludge while firing from loading to unloading, causing insufficient heat transfer rate, limited technological opportunities and complexity of operation, large size in length [23-24]. These disadvantages can be eliminated by providing a horizontal axis of rotation of the body, which will increase the speed of its rotation and simplify the operation of the furnace due to the lack of incline of the body.

Over the past ten years, we proposed design solutions to solve this problem [1-9] and provide the opportunity to implement them into production.

To move the particles of the raw material mass in the cement clinker production, technologies and equipment are used, in which cylindrical bodies tilted towards unloading are used as working bodies. The consequence of this is: significant energy costs due to the large size, limited technological capabilities, complexity of operation and a large mass of rotary kilns. To eliminate these shortcomings, technologies and equipment based on screw bodies with a horizontal axis of rotation are proposed, which allows outlining and implementing an innovative way of development of cement production, reducing energy costs, improving technical and economic indicators [10-22].

For a better representation of screw bodies, we will show their visual images made using the software package "Compass-3D".

To implement the proposed technologies and designs of rotary kilns based on screw bodies, it is necessary to create a methodology for their calculation, which allows calculating the main parameters, including one of the main ones - the speed of longitudinal movement of cement clinker granules. For example, the considered screw body of conditionally cylindrical shape is made in the form of a tetrahedral body, has the peculiarity that the vertices of the tetrahedrons form a pronounced three-way cylindrical helical line. When the screw body rotates, the clinker granules make a complex spatial movement along three screw lines, continuously passing along its walls. If you set an average speed of movement and mixing time (when moving with this speed clinker granules along the axis of rotation of the screw body), it is possible to estimate the geometric parameter of the screw body as its length $\mathrm{L}_{\text {s.b. }}$.

The inclination angle of the generative screw line relative to the axis of the screw body $\mathrm{j}=$ Const, and the edges of the tetrahedrons are secant of this line. Therefore, to study the process of transportation and mixing of clinker particles in a rotary kiln, its performance, it is necessary to specify such a parameter as the edge length of the tetrahedron " $a$ ", and then to classify the parameters under consideration:
1. The constant angular parameters of the tetrahedral screw body, which determine the spatial geometry of the moved clinker granules, where $\mathrm{j}=$ Const sets the main direction of this movement, and therefore is the main angular parameter.

2. Variable linear parameters:

$a$ - the edge length of the tetrahedron;

$\mathrm{L}_{\text {s.b. }}$ - the length of the screw body.

These parameters are complex functions of the state of kinematic parameters of clinker granules movement, transport and mixing time $t_{\text {mix }}$.

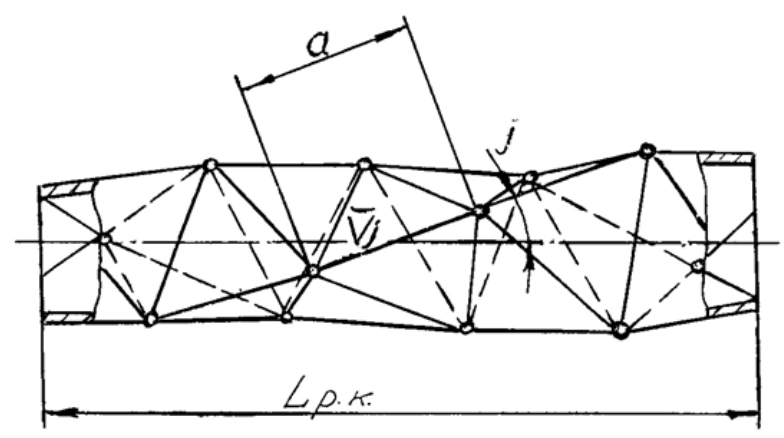

Figure 1. Kinematic $\overline{\mathrm{V}}_{\mathrm{j}}$ and geometric characteristic j, $a$ of tetrahedral screw body

\section{Methods and Materials}

Based on the nature of the technological process of transportation and mixing of raw material particles (clinker granules) in a rotary kiln during transportation and mixing $t_{\text {mix }}$ should be optimal that, of course, complicates the task of determining the parameters $\mathrm{L}_{\text {s.b. }}$, and therefore the size of the screw body, as the problem to optimize the process of transportation and mixing of raw material particles (clinker granules) as a whole should be solved.

To identify the qualitative side of the process, the problem of determining the parameter $\mathrm{L}_{\text {s.b. }}$ can be narrowed down to the following: varying the parameters a and the time of transportation and mixing of clinker granules $-t_{\text {mix }}$, it is necessary to simulate the parameters of some conditional (under certain assumptions) motion of clinker granules in order to obtain analytical expressions to describe this motion. A characteristic feature of the real movement of clinker granules is that in the absence of a pressure difference from some extraneous external sources at the input-output of the screw body, the movement will be caused by one active force of gravity in the direction of the screw lines. On the other hand, the movement that we are studying will be affected by the so- called contact forces-the forces formed as a result of collision and rolling, as well as the friction between the moving clinker granules with the surface of the rotating screw body. It should be borne in mind that the contact forces are 
involved in the direct process of transportation and mixing, determine the mixing time $t_{\text {mix, }}$ as the intensity of their impact depends on the angular velocity of rotation of the screw body. The contact forces have two ways expressed nature:

- directivity, where they participate together with gravity in the complex spatial motion of clinker granules, forming a total effect;

- stochasticity, where along complex spatial trajectories contacts (rolling and collisions) are random.

Of course, it is quite difficult to distinguish this nature, but nevertheless it is necessary, from the point of view of studying the real process of transportation and mixing in two directions: kinematic and technological.

In conclusion, it should be noted that the complex spatial motion of clinker granules is determined by the angular parameters during the rotation of the screw body the rather complex spatial geometry of the angles formed by the edges and faces of the attached tetrahedrons, among which the inclination angle of the generative screw line $\mathrm{j}=$ Const to the axis of rotation of the screw body should be considered basic, because it determines the nature of the movement of clinker granules along the three screw lines (let us imagine that we, purely abstractly, managed to reduce $\mathrm{j} \rightarrow 0$ to zero), then with such a screw line there would be no longitudinal movement. For example, if we consider the rotating cylindrical surface of the screw body, then whatever forces act in the direction perpendicular to the axis of rotation, there would not be any longitudinal displacement, as for a rotating cylinder $\mathrm{j}$ $\rightarrow 0$.

\section{Results and Discussion}

If in space (figure 1) the right triple of unit vectors $\bar{i}, \bar{j}$, $\bar{\kappa}$ are set, where the vector multiplication rule $\bar{\kappa}=\bar{i} \cdot \bar{j}$ determines the direction of rotation of the angular velocity vector of the screw body $\bar{\omega}=\omega \cdot \bar{\kappa}$, then (as established from experiments) the direction of movement along the axis of rotation of the screw body of clinker granules $M$ occurs in the opposite direction, i.e. in the direction $-\bar{\kappa}$. Thus, if the vectors $\bar{i}, \bar{j}, \bar{\kappa}$ build respectively the axis of the spatial fixed coordinate system XYZ, the components of the projections of the longitudinal displacement of the velocity $\mathrm{V}_{\mathrm{z}}<0, \mathrm{Z}<0$.

The direction $\bar{V}_{\mathrm{j}}$ is determined from experiments, $\varphi$ is the angle of rotation of the screw body.

Based on the above, it is advisable to study the process of movement of clinker granules along the direction of screw lines, given by the inclination angles $\mathrm{j}=$ Const, to investigate the behavior of some characteristics, namely the value of the speed of movement of clinker granules, for example, $\mathrm{V}_{\mathrm{j}}$ (figure2).

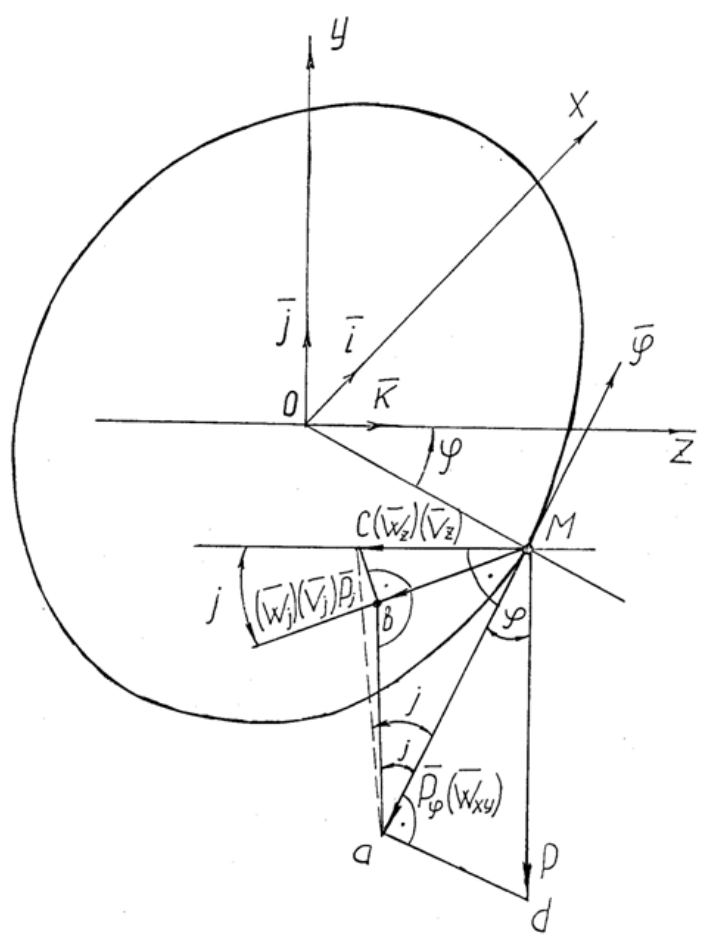

Figure 2. Scheme of action of forces on a clinker granule in screw body

Then, as it is not difficult to see (figure 3), another characteristic will be determined:

$$
\mathrm{V}_{\mathrm{j}}=\mathrm{V}_{\mathrm{Z}} \cdot \operatorname{Cos} \mathrm{j}
$$
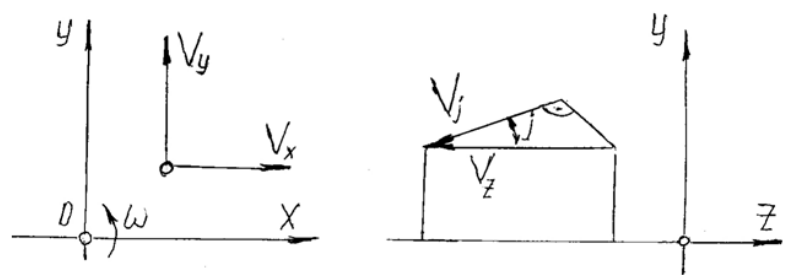

Figure 3. The scheme of directions of velocity vectors of clinker granules at their movement inside the screw body.

For a more detailed analysis of the process of clinker granules movement in a rotary kiln for cement clinker making, we introduce a simplified model of "conditional displacement": when the screw body $\omega$ is rotated, the conditional material point $\mathrm{M}$ (clinker granule) passes under the influence of forces (active gravity), successively from one edge of the tetrahedron (screw line) to another (subsequent screw line), rolls down the screw lines guides, i.e., gets the displacement (along alternating screw lines), which we will assume according to this conditional model to be continuous (in time). Then all continuous conditional displacements occur at $\mathrm{j}$ inclination angles relative to $\varphi$ geometric axis of rotation of the screw body and if the acceleration vector $W_{x y}$ (in the planes of cross sections) of the plane $\mathrm{XYZ}$ is known, then some acceleration $\mathrm{W}_{\mathrm{j}}$ of displacement along the directions of the screw lines is formed (depending on the value of $\mathrm{j}-$ inclination angle; obviously at $\mathrm{j}=0$ there would be no 
displacements), which can be written:

$$
\overline{W_{j}}=W_{x y} \operatorname{Sin} j,
$$

where $\mathrm{W}_{\mathrm{j}}=\frac{\mathrm{dVj}}{\mathrm{dt}}$.

This formula follows from simple physical considerations.

Vector-component of the weight force:

$$
\overline{\mathrm{P}}_{\varphi}=-\overline{\mathrm{P}} \operatorname{Cos} \varphi
$$

will be projected on the $\mathrm{j}$ - direction by the formula

$$
\overline{\mathrm{P}}_{\mathrm{j}}=\overline{\mathrm{P}}_{\varphi} \cdot \operatorname{Sinj}
$$

Similarly, the mass forces of friction and collisions will be projected according to the same dependence.

According to Newton's $2^{\text {nd }}$ law

$$
\overline{\mathrm{W}}_{\mathrm{xy}}=\frac{1}{\mathrm{~m}} \sum_{\mathrm{i}=1}^{\mathrm{n}} \overline{\mathrm{F}}_{\mathrm{I}},
$$

where $\overline{\mathrm{F}}_{\mathrm{I}}$ includes the component of the weight force $\overline{\mathrm{P}}_{\varphi}$ :

$\varphi$ - the direction of rotation of the point $\mathrm{M}$ (clinker granules) in the XOY plane;

$\mathrm{P}$ - the weight of the clinker granule;

$\mathrm{P} \varphi$ - component $\mathrm{P}$ on $\varphi$-direction,

$\mathrm{P} \varphi-\mathrm{P} \cdot \operatorname{Cos} \varphi-$ the value of this component per direction $\varphi$.

Figure 1 shows that ав $\perp \mathrm{MB}, \mathrm{aM} \perp 0 \mathrm{z}$, therefore $\angle 8 a M$ (by virtue of mutually crossing pairs: $O Z$ and $\mathrm{BM}, \mathrm{Ma}$ of straight segments).

Therefore, the entire vector $\bar{W}_{\mathrm{xy}}$ will be projected to the direction by the dependence:

$$
\overline{W_{j}}=W_{x y} \cdot \operatorname{Sin} j
$$

Let us note that if we are interested in continuous movement (conditional) along the axis of rotation (in the direction opposite to the choice of the $\mathrm{Z}$ axis, figure1), we can write:

$$
\overline{\mathrm{W}}_{\mathrm{z}}=\dot{\mathrm{V}}_{\mathrm{z}}=\overline{\mathrm{W}}_{\mathrm{xy}} \cdot \operatorname{tg} \mathrm{j},
$$

where $\mathrm{W}_{\mathrm{z}}=\frac{\mathrm{Vz}}{\mathrm{dt}}$.

The relation (7) follows directly (figure 1) from the condition:

$$
\mathrm{cM}=\mathrm{aM} \cdot \operatorname{tg} \mathrm{j}
$$

Relations (2) and (7), their compatibility should be interpreted in this model of "conditional movement" as follows:

Let the vectors $\overline{\mathrm{W}}_{\mathrm{z}}, \overline{\mathrm{W}}_{\mathrm{xy}}$ be projected in such a way on the j-direction that the condition is met:

$$
\overline{\mathrm{W}_{\mathrm{J}}}=\mathrm{W}_{\mathrm{xy}} \cdot \operatorname{Sin} \mathrm{j} \simeq \overline{\mathrm{W}}_{\mathrm{z}} \cdot \operatorname{Cosj}
$$

From these two relations (conditional to some extent) immediately (after integration), as it is easy to see, another characteristic will be determined (figure 4) i.e., a connection will be established between the values of the displacement velocities under the condition $\mathrm{t}_{0}=0, \mathrm{~V}_{\mathrm{j} 0}=$ $\mathrm{V}_{\mathrm{z} 0}$ for the initial conditions.

$$
V_{j}=V_{z} \cdot \operatorname{Cos} j \text { or } V_{z}=\frac{V j}{\operatorname{Cos} j} .
$$

Let us note that $V_{j}<V_{z}$ which follows from the expression (10), is a condition of "shortest movement" along the direction of screw lines.

Thus, for $\rho=r=r_{c p}(\alpha)=$ const the resulting conditional model is a special case of a crew line having, as it is known, the following properties:

1. The tangents (in this case, the $V_{j}$ vector) form a constant angle (meaning $\mathrm{j}=$ Const) with the axis of the screw body.

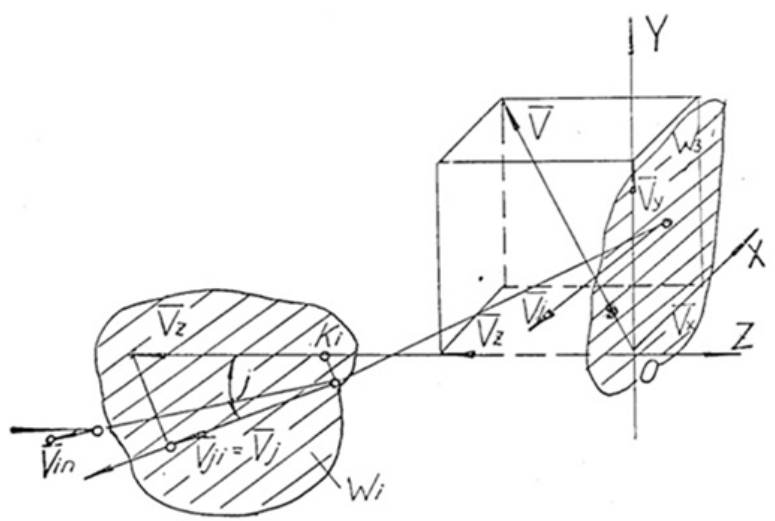

Figure 4. The layout of the planes of action of forces on the clinker granule in the screw body

2. The shortest distance (in our case, moving) between two points on the cylinder along a screw line passes through these points (this property, perhaps, reflects $\mathrm{V}_{\mathrm{j}}<$ $\mathrm{V}_{\mathrm{z}}$ in our conditional model).

Thus, the process studied (simulated conditionally) by us in figures 1 and 4 should be investigated:

- in cross-sectional planes X0Y $\left(\mathrm{W}_{3}\right)$,

- in planes of instant spins $\mathrm{W}_{\mathrm{i}}$, where $\overline{\mathrm{V}}_{\mathrm{Z}}$ i designed for constant angles on $\bar{V} i, j$ - the direction of the vectors: $\mathrm{V} \mathrm{i,j}$ are the tangent vectors in each point of the screw line through the vector $\mathrm{V} \mathrm{j}$ and the point $\mathrm{K} \mathrm{i}$, lying on the axis of rotation is the instantaneous plane $\mathrm{W}_{\mathrm{i}}$ :

$$
\mathrm{V}=\sqrt{\mathrm{V} \frac{2}{\mathrm{x}}+\mathrm{V} \frac{2}{\mathrm{y}}+\mathrm{V} \frac{2}{\mathrm{z}}}
$$

The dependence (10) is a specific feature of the studied real motion and mixing of clinker granules, as it is included in the expression for the resulting value of their velocities.

Modeling of the real process of movement of clinker granules should be carried out taking into account these features:

- gravity, contact forces (interaction of clinker granules) during the rotation of the screw body and depending on the cross-sectional profile of the screw body cause rotational and translational motion in the plane $\mathrm{XOY}$; gravity as the active force directed perpendicular to the axis of rotation of the screw body causes the 
clinker granules to roll along the screw lines in the opposite direction of the axis;

- the motion in the $\mathrm{j}$ direction becomes generally known in the study of motion in the plane of rotation $\mathrm{XOY}\left(\mathrm{W}_{3}\right)$, as it is only necessary to design the forces considered in this plane on the $\mathrm{j}$ direction (it is expressed); the $j$ direction should be understood as the direction of the studied motions along the vectors $\bar{V}_{j} i$;

- it should be noted that the definition of the nature of contact forces remains somewhat difficult when designing on the $\mathrm{j}$ direction.

When modeling, in order to obtain analytical expressions, it is necessary to somehow "roughen" the contact forces, as well as the geometry of the cross-section profile of the screw body, hence the accuracy of the studied characteristics. And the accuracy depends on the ratio of the values of the projections on the $j$ direction of gravity and the resultant directed from the contact forces. Modeling can be carried out by studying the continuous motion of all clinker granules as a whole, as well as individually, studying their continuous motion, for example, each clinker granule. An obvious assumption (coarsening) when considering these two models is the essential condition:

- in the first model, clinker granules are considered as a continuous medium with some conditional variable density of mass distribution of material points;

- in the second model, clinker granules are considered as material points of different weight.

According to the solid medium model, the most important vector characteristic is the velocity $\mathrm{V}(\mathrm{x}, \mathrm{y}, \mathrm{z}, \mathrm{t})$, the value of which should be taken into account, taking into account the expressions (10) and (11), and their features. This function characterizes the flow rate of clinker granules at each fixed point in the space of XYZ coordinate system at time $t$ and gives information about the movement as a whole. The main condition for the application of this model is that at each point of the volume forces must be directed along the normal to the surface, tightening the volume of clinker granules. Contact forces in the considered model should be superficial, short-acting. Using the apparatus of transformation of surface integrals into volume integrals (Ostrogradsky-Gauss formulas) it is possible to transform these forces, to come to the equations of motion of a continuous medium, linking the characteristics of motion with thermodynamic characteristics. The right parts of these equations will contain partial derivatives and obtaining analytical expressions when integrating them can be clearly difficult.

Therefore, it makes sense to consider the second model first in order to identify certain qualitative features of the simulated process.

Let each moving clinker granule be some conditional material point of mass $\mathrm{m}$, participate in the XOY plane in rotational-translational motion depending on the cross-sectional profile of the screw body (figure 5). Then, introducing the polar coordinate system, we write in projections on the coordinate axes:

$$
\left\{\begin{array}{l}
x=\rho \cdot \operatorname{Cos} \varphi ; \\
y=\rho \cdot \operatorname{Sin} \varphi
\end{array}\right.
$$

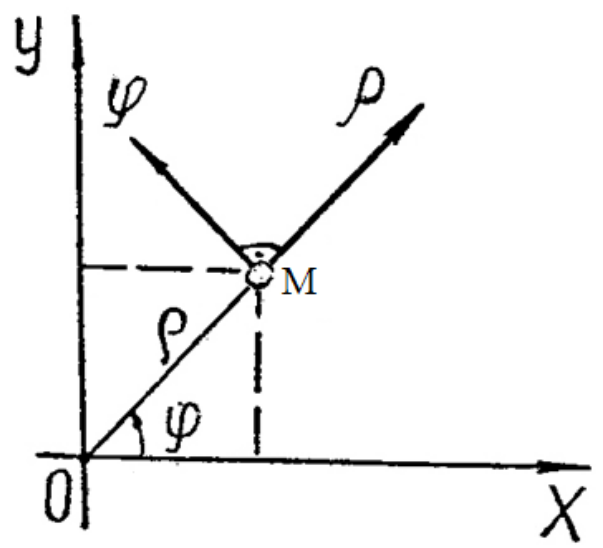

Figure 5. Scheme of motion of a clinker particle in the XOY plane of a screw body

The equation of motion (12) can be used to determine $\mathrm{V}_{\mathrm{x}}, \varphi, \mathrm{V}_{\mathrm{y}}$, and therefore to determine $\mathrm{V}_{\mathrm{x}}^{2}+\mathrm{V}_{\mathrm{y}}^{2}$ when substituting into expression (11). In carrying out further analytical research, if you design the contact forces and gravity $\rho, \varphi$ in the direction of change of the rotation radius and rotation angle, respectively, it is possible to write the known differential equations of motion of the material point $M$ (clinker granules) and after the simplification $\rho=r=r_{c p}(\alpha)=$ const, we obtain an analytical dependence for determining the longitudinal velocity of the clinker granules in the screw body of the rotary kiln in the form of:

$$
\mathrm{V}_{\mathrm{z}}=\mathrm{r} \cdot \operatorname{tgj} \cdot \omega+\mathrm{C} .
$$

where $\mathrm{C}$ - constant function $\mathrm{K}_{\mathrm{v}}$ - the fill factor of the screw body and $\theta$ - the inclination angle of the axis of rotation of the screw body.

The conducted researches allowed receiving analytical dependence for calculation of speed of longitudinal movement of granules of cement clinker in the screw body of the rotating furnace. It should be assumed that the features of the proposed designs of the screw body include the fact that the inner perimeter of the screw body not only broken or smooth screw lines, but also the screw surface along their entire length are formed, which provides a violation of the stationary flow of particles of raw cement mass, increase productivity and expansion of technological capabilities. Based on the nature of the technological process of transportation and mixing of raw material particles (clinker granules) in a rotary kiln after selecting the time of transportation and mixing $t_{\text {mix }}$ it is possible to determine by means of the received dependence longitudinal speed of movement of cement 
clinker granules, consequently the sizes of the screw body.

\section{Conclusions}

The use of the proposed designs of screw bodies, in comparison with the known designs of similar purpose, increases productivity significantly, reduces the size, weight of rotary kilns and energy consumption. The technical solutions protected by patents of the Russian Federation are offered; the visual images of some designs of screw bodies of the rotating furnaces executed with use of a software complex "Compass-3D" are shown. The analytical dependence for determining the longitudinal velocity of clinker granules in the screw body of the rotary kiln is obtained. The conducted researches open possibilities of wide implementation of innovative technologies of cement clinker making on the basis of screw bodies of the rotating furnaces.

\section{REFERENCES}

[1] Aleksandr Sekisov, Georgy Serga, "Cement kiln", Pat. 2692141 Russian Federation, IPC F27B 7/14, Kuban State Technological University, No. 2018144137, declared 12.12.2018, publ. 21.06.2019, Bul. No $18-3$ p.

[2] Aleksandr Sekisov, Georgy Serga, "Rotary kiln for the preparation of cement clinker", Pat. 2694181 Russian Federation, IPC F27B 7/16, Kuban State Technological University, No. 2018144138, declared 12.12.2018, publ. 09.07.2019, Bul. No $19-3$ p.

[3] Victor Taratuta, Kirill Belokur, Georgy Serga, "Rotary kiln for preparation of cement clinker (options)", Pat. 2456520 Russian Federation, IPC F27B 7/16, Kuban State Agrarian University, No. 2009126872/03; declared 13.07.2009; publ. 20.01.2011, Bul. No 20. -3 p.

[4] Georgy Serga, Victor Taratuta, "Rotary kiln for the preparation of cement clinker", Pat. 2536318. Russian Federation, IPC F27B 7/16, Kuban State Agrarian University, No 2013120422/02; declared 30.04.2013; publ. 20.12.2014, Bul. No 35. 3 p.

[5] Victor Taratuta, Georgy Serga, "Cement kiln", Pat. 2583215. Russian Federation, IPC F27B 7/14, Kuban State Agrarian University. No 2014144250/02; declared 31.10.2014; publ. 10.05.2016, Bul. No 13. - 3 p.

[6] Victor Taratuta, Georgy Serga, "Rotary kiln for sludge firing for cement clinker preparation”, Pat. 2561571. Russian Federation, IPC F27B 7/14, Kuban State Agrarian University, No 2014114567/02; declared 11.04.2014; publ. 27.08.2015, Bul No 24. -3 p.

[7] Victor Taratuta, Georgy Serga, "Furnace for the preparation of cement clinker", Pat. 2533292. Russian Federation, IPC F27B 7/16, Kuban State Agrarian University, No 2013120415/02; declared 30.04.2013; publ. 20.11.2014, Bul. No 2. -3 p.
[8] Kirill Belokur, Georgy Serga, "Cement kiln (options)", Pat. 2483260 Russian Federation, IPC F27B 7/14, Kuban State Agrarian University, No. 2009143193/03; declared 23.11.2009; publ. 27.05.2013, Bul. No 15. - 3 p

[9] Victor Taratuta, Kirill Belokur, Georgy Serga, "Rotary kiln for the preparation of cement clinker", Pat. 2476793 Russian Federation, IPC F27B 7/14, Kuban State Agrarian University, No 2011106741/02; declared 22.02.2011; publ. 27.02.2013, Bul. No 6. -3 p

[10] Aleksandr Sekisov, "Machines based on combined rotors in mechanical engineering", Intern. Col. of Scient. Works Progressive technologies and systems of mechanical engineering, DONNTU-Donetsk, № 3(66), PP.69-80, 2019

[11] Aleksandr Sekisov, Georgy Serga. "Rotary-screw systems for rotary kilns", E3S Web of Conferences, Topical Problems of Architecture, Civil Engineering and Environmental Economics (TPACEE-2018), Vol. 91, 02034, 2019.

Https://www.e3s-conferences.org/articles/e3sconf/pdf/201 9/17/e3sconf tpacee2019 02034.pdf, doi:https://doi.org/10.1051/e3sconf/20199102034.

[12] Rinad Popov, Natalia Shipilova, Aleksandr Sekisov, Yekaterina Solovyova, Dmitry Gura, "Innovative development of construction in russia: economics, technologies, management", Amazonia Investiga, Colombia, Vol. 8, № 19, 2019, PP. 653-663. Https:/amazoniainvestiga.info/index.php/amazonia/article/ view/281/258.

[13] Valery Lebedev et al, "Increase of efficiency of finishing-cleaning and hardening processing of details based on rotor-screw technological systems", IOP Conf. Ser.: Mater. Sci. Eng. 327 042062, 2018. DOI: https://doi.org/10.1088/1757-899X/327/4/042062.

[14] Valeriy Lebedev, Georgy Serga, Irina Davydova, Tatiana Atoyan, Irina Koshlyakova, Alexander Gordienko, "Method for calculating the power of a rotor-screw machines", MATEC Web Conf., 226 01007, 2018. DOI: https://doi.org/10.1051/matecconf/201822601007.

[15] Valeriy Lebedev, Serga, Irina Davydova, Tatiana Atoyan, Irina Koshlyakova, Alexander Gordienko, "Main trends in intensification of rotor-screw processing of parts", MATEC Web Conf., 226 01008, 2018. DOI: https://doi.org/10.1051/matecconf/201822601008.

[16] Alexey Marchenko, Georgy Serga, "Creating a Methodology for Calculating the Drive of the Working Parts of the Equipment Based on the Original Screw Sieves, Screw Housings and Screw Drums", Research journal of pharmaceutical biological and chemical sciences, Volume 10, Issue 1. PP: 1689-1696, Publ: JAN-FEB 2019.

[17] Alexey Marchenko et al., "Creating a Methodology for Calculating the Drive of the Working Parts of the Equipment Based on the Original Screw Sieves, Screw Housings and Screw Drums", Indo Am. J. P. Sci, 06(03), 2019.

[18] Alexey Marchenko et al., "Investigation of Contact Forces of Interaction of Particles of Bulk Materials During their Movement in Helical Drums", Indo Am. J. P. Sci, 06(03), 2019. 
[19] Victor Butenko, Valery Lebedev, Irina Davydova and Georgy Serga, "Abrasive tool for processing components made from chrome-nickel steels and alloys", E3S Web Conf., $11001040,2019 .$.

[20] Rinad Popov, Aleksandr Sekisov, Natalia Shipilova, "The Economics of Innovation in Modern Russia: Practice, Problems and Prospects", International Journal of Economics and Financial Issues, Vol. 6, Special Issue (S8), PP.184-188,

2016.

Https:/www.econjournals.com/index.php/ijefi/article/view /3727/pdf.

[21] Rustam Nehaj, Georgij Molotkov, Ivan Rudchenko, Anatolij Grinev, Aleksandr Sekisov, "Algorithm of composing the schedule of construction and installation works", IOP Conference Series: Earth and Environmental
Science, Vol. 90, Issue 1 PP.1-8, 2017.

[22] Aleksandr Sekisov et al., "Development of the Methods Improving the Production Costs Formation Process", Materials Science Forum, Vol. 931, PP. 1210-1213, 2018.

[23] Trong-Phuoc Huynh, Van-Hien Pham, Tri-Khang Lam , Nguyen-Trong Ho, "Experimental Research on the Performance of Polypropylene Fiber Foamed Ultra-lightweight Composites," Civil Engineering and Architecture, Vol. 8, No. 4, pp. 654 - 661, 2020. DOI: 10.13189/cea.2020.080429.

[24] Quoc Gia Hoang, Quoc Vuong Vu, "Estimation the Initial Cement Dosage of Concrete from Mechanical Behavior and Chemical Analysis," Civil Engineering and Architecture, Vol. 8, No. 4, pp. 580 - 585, 2020. DOI: $10.13189 /$ cea.2020.080422. 